\title{
A dual-functional ferroferric oxide/quantum dots theranostic nanoplatform for fluorescent labeling and photothermal therapy
}

Naiqiang Yin ( $\nabla$ yinnq@foxmail.com )

Shangqiu Normal University https://orcid.org/0000-0002-4615-3999

Xianling Wang

Shangqiu Normal University

Tonghui Yang

Shangqiu Normal University

Yanli Ding

Shangqiu Normal University

Liqiang Li

Shangqiu Normal University

Peng Li

Shangqiu Normal University

Xiaoliang Xu

University of Science and Technology of China

Lixin Zhu

Anhui Medical University

\section{Research Article}

Keywords: theranostic nanoplatform, mesoporous silica nanoparticles, quantum dots, photothermal therapy, fluorescent labeling

Posted Date: March 10th, 2021

DOl: https://doi.org/10.21203/rs.3.rs-294211/v1

License: (1) This work is licensed under a Creative Commons Attribution 4.0 International License. Read Full License

Version of Record: A version of this preprint was published at Particle \& Particle Systems Characterization on April 30th, 2021. See the published version at https://doi.org/10.1002/ppsc.202100043. 


\section{Abstract}

A biocompatible, nontoxic theranostic nanoplatform consisting of mesoporous silica-coated ferroferric oxide $\left(\mathrm{Fe}_{3} \mathrm{O}_{4}\right)$ and $\mathrm{Mn}$-doped $\mathrm{ZnS}-\mathrm{ZnS}$ quantum dots has been synthesized via a layer-by-layer method. Transmission electron microscopy, X-ray diffractometer, magnetometry, and fluorophotometer were employed to characterize the nanoplatform. The nanoplatform exhibited excellent superparamagnetic, fluorescent and light adsorption properties. A template method was introduced to form a mesoporous silica structure on the nanoplatform, lowering the mass of the nanoplatform and effectively promoting the absorption of incident light, compared with traditional silica layer. In addition, after endocytosis of the nanoplatform, cancer cells were easily detected under fluorescence microscope because of the excellent fluorescent behavior of quantum dos. Moreover, vitro cell experiments confirmed that nanoplatform possessed perfect photothermal effect to destroy tumor cells under the laser irradition. Therefore, ferroferric oxide/quantum dots nanoplatforms, combined with fluorescent labeling and photothermal therapy for cancer cells, are expected to be a promising biopotential materials in the field of diagnosis and treatment.

\section{Introduction}

With the rapid development of science and technology, nanomedicine materials had gain extensive attention and been applied in various medical diagnosis and treatment, such as photothermal therapy ${ }^{[1}$, ${ }^{2]}$, chemodynamic therapy ${ }^{[3-5]}$, fluorescent labeling ${ }^{[6,7]}$ and magnetic resonance imaging ${ }^{[8]}$. Ferroferric oxide $\left(\mathrm{Fe}_{3} \mathrm{O}_{4}\right)$ nanoparticles, possessing the advantage of easy-synthesis process and environmental friendliness, were pursued and analyzed by biomedical scientists in recent years ${ }^{[9-11]}$. Photothermal effect, as a typical and outstanding property of $\mathrm{Fe}_{3} \mathrm{O}_{4}$ nanoparticles, was a photothermal conversion process, during which the irradiation light energy was absorbed by the nanoparticle and transformed into local hyperthermia ${ }^{[12-14]}$. As the nanoparticle was transported to targeted cancer cells, the local hyperthermia would generate high temperature and lead to the cancer cell inactivation. Gupta group reported that eugenate capped $\mathrm{Fe}_{3} \mathrm{O}_{4}$ nanoparticles exhibited a distinct light absorption behavior and were successfully employed to destroy the deep tissue based on the phototheral therapy ${ }^{[15]}$. Besides, the $\mathrm{Fe}_{3} \mathrm{O}_{4}$ nanoparticle with the size of smaller than $20 \mathrm{~nm}$ exhibits excellent superparamagnetic behavior. Under high gradient external magnetic field, drug molecules banded onto the biocompatible $\mathrm{Fe}_{3} \mathrm{O}_{4}$ nanoparticles could be easily delivered to the target region ${ }^{[16]}$.

Coprecipitation method and organometallic decomposition method were the main approach to obtain high-quality $\mathrm{Fe}_{3} \mathrm{O}_{4}$ nanoparticles ${ }^{[17,18]}$. Though the organometallic decomposition method played a significant role to prepare a better $\mathrm{Fe}_{3} \mathrm{O}_{4}$ nanoparticle with perfect crystal texture and high monodispersity, the hydrophobic property of the obtained nanoparticles seriously restricted the biomedical application of delivery and dispersion in vivo experiment. Due to the intrinsic magnetism, the $\mathrm{Fe}_{3} \mathrm{O}_{4}$ nanoparticles tend to attract each other and produce the phenomenon of agglomeration, leading to 
the occurrence of precipitation. Therefore, a further decoration on the $\mathrm{Fe}_{3} \mathrm{O}_{4}$ nanoparticles was a necessary step to ensure them good biocompatibility and stable circulation in the biomedical applications. In the previous reports ${ }^{[19-21]}$, silica $\left(\mathrm{SiO}_{2}\right)$, as a suitable inter-carrier, not only could be easily modified onto the surface of $\mathrm{Fe}_{3} \mathrm{O}_{4}$ nanoparticles to avoid the appearance of precipitation, but also could be functioned with different groups to connect with other nanomaterials. Besides, it was well-known that the irradiation light could be strongly absorbed or scattered by encountered medium, resulting in the reduction of irradiation light intensify. A mesoporous structure of silica was a suitable substitution to lower the density of silica and enhance the transmissivity of irradiation light, avoiding the side effect of absorption and scattering ${ }^{[22,23]}$.

In the field of disease diagnosis, positron emission tomography, magnetic resonance imaging, optical imagings were the primary imaging techniques for noninvasive detection in vivo ${ }^{[24,25]}$. Among them, great interest had been devoted to the optical imaging technology due to its high resolution, easy operation, and low cost. Superior to the organic fluorescence dye, quantum dots (QDs) possessed many promising physicochemical properties, including size-dependent emission bands, superior photostability and larger Stokes shifts ${ }^{[26-28]}$. However, the problem of the intrinsic toxicity of traditional Cd-based quantum dots seriously limited their further clinical use. In order to solve the toxicity restriction, new type non-toxic Mn-doped ZnS QDs, consisting of Mn ion as the emission center and ZnS as the host, were introduced by scientists in the past few years. Another advantage about the Mn-doped ZnS QDs was that the emission behavior was not seriously affected by the relatively poor crystal quality. Recently, our group reported a Mn-doped ZnCdS/ZnS core/shell QDs, possessing tunable excitation wavelength and bright emission wavelength, were successfully employed to label the targeted cancer cell.

Though individual $\mathrm{Fe}_{3} \mathrm{O}_{4}$ nanoparticle and quantum dots exhibited excellent photothermal effect and perfect fluorescence behavior respectively, there was still much space to engineer a new complex nanostructure by integrating the $\mathrm{Fe}_{3} \mathrm{O}_{4}$ nanoparticle and quantum dots. Here, we designed a theranostic nanoplatform, decorating Mn-doped $\mathrm{ZnS} / \mathrm{ZnS}$ QDs onto the surface of mesoporous $\mathrm{SiO}_{2}$ coated $\mathrm{Fe}_{3} \mathrm{O}_{4}$ nanoparticle based on the electrostatic interaction for the first time. During the coating progress of $\mathrm{SiO}_{2}$ onto $\mathrm{Fe}_{3} \mathrm{O}_{4}$ nanoparticle, larger pores on the internal $\mathrm{SiO}_{2}$ layer were formed relying on synergistic effect of the template method and pore-enlarging agent. The as-synthesized nanoplatform exhibited superparamagnetism, and could easily dragged to the direction of the external magnet. In addition, the biological experiments demonstrated that the endocytosis of the nanoplatform by cancer cells was efficient, which could be confirmed by the successful detection of the cancer cells under fluorescence microscope. Furthermore, combining with the treatment of the nanoplatform and irradiation exposure, irreversible destruction to the cancer cells was readily examined. In conclusion, the nanoplatform realized simultaneous magnetic separation, fluorescence imaging, and photothermal therapy. We believed that such a compositive nanoplatform had the potential to successfully challenge some of the currently met limitations in medical diagnosis and treatment. 


\section{Experimental \\ 2.1 Materials}

zinc acetate (99\%), Ferric chloride (98\%), Iron( $(\mathbb{)})$ sulfide (99\%-Fe), Ammonia solution ( $28 \%$ in water), ethanol (99.8\%), sodium hydroxide ( $\mathrm{NaOH}, 97 \%)$, oleic acid(99\%), tetraethoxysilane (TEOS, 98\%), hexadecyltrimethyl ammonium bromide (CTAB, 98\%), 1, 3, 5-trimethyl-benzene (TMB, 99\%), chloroform(99.8\%), ethylene glycol (EG, 99.5\%), and folic acid (96\%) were purchased from Sinopharm Chemical Reagent Co., Ltd, China. 3-Mercaptopropionic acid (MPA, 99.0\%), sodium sulfide nonahydrate (99.0\%), and 3-aminopropyltrimethoxysilane (APS, 97\%) were ordered from Sigma-Aldrich. Breast cancer cells (MDA-MB-231) were provided Anhui Medical University. The other reactants and solvents were of analytical grade without further purification.

\subsection{Synthesis of $\mathrm{Fe}_{3} \mathrm{O}_{4}$ nanoparticle}

Chemical coprecipitation method was introduced and employed to prepare $\mathrm{Fe}_{3} \mathrm{O}_{4}$ nanoparticle, possessing the advantages of being simple, environmental friendly and high-yield. Typically, Ferric chloride (3g), Iron( $(\mathbb{)})$ sulfide $(1.7 \mathrm{~g})$ and $20 \mathrm{~mL}$ deionized water were loaded into a $50 \mathrm{~mL}$ three necked round-bottom flask and degassed at room temperature for $30 \mathrm{~min}$ under nitrogen flow. Then the temperature was heated to $80^{\circ} \mathrm{C}$ with the protection of nitrogen. Concentrated ammonia solution was added to regulate the $\mathrm{pH}$ to 9.8. To the mixture solution, $1 \mathrm{~mL}$ oleic acid was added dropwise under vigorous stirring for the decoration the nanoparticles. After completion of modification process for 2 hours, the obtained $\mathrm{Fe}_{3} \mathrm{O}_{4}$ nanoparticles were repeatedly purified with deionized water and stored in 20 $\mathrm{mL}$ chloroform for further characterization and use.

\subsection{Preparation of Mn-doped $\mathrm{ZnS} / \mathrm{ZnS}$ quantum dots}

The preparation of Mn-doped ZnS/ZnS QDs was performed through a simple liquid phase reaction process according to our previous report ${ }^{[29]}$. Also, the stock solutions were prepared by dissolving the precursor in a certain amount of deionized water. $5 \mathrm{ml}$ zinc acetate $(0.1 \mathrm{M}), 1.5 \mathrm{ml}$ manganese acetate $(0.01 \mathrm{M})$ and $40 \mathrm{ml}$ MPA $(0.05 \mathrm{M})$ were loaded into a $100 \mathrm{~mL}$ three necked round-bottom flask under vigorous stirring and degassed for 30 minutes in nitrogen atmosphere. The $\mathrm{pH}$ value of the solution was adjusted to 10.3 with drops of $1 \mathrm{M} \mathrm{NaOH}$ with stirring. After bubbling for another 30 min under nitrogen flow, $4.5 \mathrm{ml}$ sodium sulfide nonahydrate $(0.1 \mathrm{M})$ was added into the solution via a peristaltic pump in a period of 2 hours. As the completion of growth, the Mn-doped ZnS nanocrystals were aged at $50^{\circ} \mathrm{C}$ for another 2 hours. Then the obtained Mn-doped ZnS quantum dots were cooled to room temperature, and purified with absolute ethanol. After redissolved in $20 \mathrm{~mL}$ deionized water, the as-prepared $\mathrm{Mn}$-doped $\mathrm{ZnS}$ QDs were heated to $100^{\circ} \mathrm{C}$ and bubbled for 10 minutes under nitrogen flow. $3.8 \mathrm{~mL}$ zinc acetate $(0.1 \mathrm{M})$ was added into crude QDs solution at intervals of 20 minutes. Immediately after refluxed for 30 minutes at $100^{\circ} \mathrm{C}$, the Mn-doped $\mathrm{ZnS} / \mathrm{ZnS}$ quantum dots purified with absolute ethanol for three times. Finally, the purified quantum dots were redissolved in $20 \mathrm{~mL}$ deionized water for further use. 


\subsection{Synthesis of $\mathrm{Fe}_{3} \mathrm{O}_{4} /$ quantum dots nanoplatform}

$1 \mathrm{~mL}$ prepared $\mathrm{Fe}_{3} \mathrm{O}_{4}$ nanoparticle, $60 \mathrm{~mL} \mathrm{CTAB}(0.1 \mathrm{M})$ and $0.5 \mathrm{~mL}$ TMB were loaded into a three necked round-bottom flask and sonicated for $30 \mathrm{~min}$ at $50^{\circ} \mathrm{C}$. After aged at $70^{\circ} \mathrm{C}$ for $30 \mathrm{~min}$, the mixture solution was added by $10 \mathrm{~mL} E G$, and $0.7 \mathrm{~mL}$ aqueous ammonia solution. Then $20 \mu \mathrm{L}$ TEOS were added into the mixture solution at intervals of 30 minutes with fast mechanical stirring. As the completion of reaction for 3 hours, the $\mathrm{SiO}_{2}$ coated $\mathrm{Fe}_{3} \mathrm{O}_{4}$ nanoparticles were purified with absolute ethanol, and excess ammonium nitrate was added into the solution at $60^{\circ} \mathrm{C}$ for disposing the $\mathrm{CTAB}$ molecule from $\mathrm{SiO}_{2}$ layer. Then the obtained $\mathrm{Fe}_{3} \mathrm{O}_{4} @$ mesoporous $\mathrm{SiO}_{2}$ nanoparticles were redissolved in $50 \mathrm{~mL}$ absolute ethanol. $0.5 \mathrm{~mL}$ APS was added dropwise into the $\mathrm{Fe}_{3} \mathrm{O}_{4} @$ mesoporous $\mathrm{SiO}_{2}$ solution obtained previously, while under vigorous stirring. As the completion of reaction for 30 minutes, excess absolute ethanol was used to wash the particles and diluted with deionized water $(40 \mathrm{~mL})$. Subsequently, the obtained $\mathrm{Fe}_{3} \mathrm{O}_{4} @$ mesoporous $\mathrm{SiO}_{2}(10 \mathrm{~mL})$ and $\mathrm{Mn}$-doped $\mathrm{ZnS} / \mathrm{ZnS}$ quantum dots $(1 \mathrm{~mL})$ were loaded into a $50 \mathrm{~mL}$ roundbottom flask at room temperature. After continuous stirring for 24 hours, the obtained products were purified by centrifugation and ultrasonic treatment. Finally, $2 \mathrm{mg}$ folic acid was dropwise added into the product under mild agitation. As the completion of the decoration for 12 hours, the $\mathrm{Fe}_{3} \mathrm{O}_{4}$ /quantum dots nanoplatform was purified by centrifugation for 3 times, and redissolved in phosphate buffer saline (PBS) solution for characterization and use.

\subsection{Cell labeling experiment}

The breast cancer cells (MDA-MB-231) were maintained at $37^{\circ} \mathrm{C}$ with $5 \% \mathrm{CO}_{2}(\mathrm{v} / \mathrm{v})$ on glass coverslip in 12-well culture plate. Immediately after the coverage reached $60-80 \%$ on the glass coverslip, the cancer cells were washed with excess PBS solution. Then $50 \mu \mathrm{L} \mathrm{Fe} \mathrm{O}_{4}$ /quantum dots nanoplatform were introduced onto the culture plate and further incubated for 2 hours at $37^{\circ} \mathrm{C}$ with $5 \% \mathrm{CO}_{2}(\mathrm{v} / \mathrm{v})$. Finally, the glass coverslip was washed with PBS solution for three times to remove the unuptaked nanoplatforms, and then the cancer cells were prepared to detect the labeling behavior under fluorescence microscopy.

\subsection{Photothermal therapy in vitro}

On the one hand, aqueous solutions of $\mathrm{Fe}_{3} \mathrm{O}_{4}$ /quantum dots nanoplatform samples were irradiated by an $808 \mathrm{~nm}$ laser with different concentration. The temperature of all samples was recorded, and all the dates were obtained from the average of five times measurement. On the other hand, the photothermal therapy for cancer cells was investaged. As harvested from the culture plate and co-incubation with adequate nanoplatform, the cancer cells were washed with excess PBS solution. Then the cancer cells were irradiated by an $808 \mathrm{~nm}$ laser with the power density of $1.0 \mathrm{~W} / \mathrm{cm}^{2}$. After irradiated for 10 minutes, the cancer cells were stained to examine the live and dead cells under fluorescence microscopy.

\subsection{Characterization}

The crystal structure was measured by X-ray diffractometer (Rigaku-Dmax 2500) with Cu Ka (0.15405 $\mathrm{nm}$ ) radiation. The morphologies were inspected on transmission electron microscope (TEM, JEM-1400) 
with acceleration voltage of $200 \mathrm{kV}$. Magnetic measurement was performed by vibrating sample magnetometer (Quantum Design) at 300K. The Photoluminescence (PL) emission spectra were examined by spectrofluorimeter (Hitachi F-4500) equipped with Xenon lamp as the light source.

\section{Results And Discussion}

Recently, great effect had been devoted to synthesize complex nanostructures to satisfy the need of multiple functions in the biomedical research. However, the disadvantage of biological toxicity severely limited the clinical application. Here, a layer-by-layer assembly was employed to design a biocompatible, nontoxic $\mathrm{Fe}_{3} \mathrm{O}_{4} / \mathrm{Mn}$-doped $\mathrm{ZnS} / \mathrm{ZnS}$ nanoplatform, which was suitable for photothermal therapy and targeted labeling for cancer cells. As described in scheme 1, the nanoplatform was prepared using $\mathrm{Fe}_{3} \mathrm{O}_{4}$ core for the photothermal therapy and effective magnetic separation, Mn-doped $\mathrm{ZnS} / \mathrm{ZnS}$ quantum dots for targeted labeling, mesoporous $\mathrm{SiO}_{2}$ for structural stability, and folic acid for good endocytosis. Due to the loose structure of mesoporous $\mathrm{SiO}_{2}$, the density of the nanoplatform was effectively lowered, and much radiation light could permeate and absorbed by the $\mathrm{Fe}_{3} \mathrm{O}_{4}$ core, exhibiting a much higher efficiency of photothermal therapy for cancer cells.

Gram-scale hydrophilic $\mathrm{Fe}_{3} \mathrm{O}_{4}$ nanoparticles were fabricated by a facile co-precipitation approach using ammonia as catalyst and oleic acid as surface stabilizer. As reported in our previous literature ${ }^{[30]}$, the hydrosoluble $\mathrm{Fe}_{3} \mathrm{O}_{4}$ nanoparticles had uniform, narrow size distribution of around $11 \mathrm{~nm}$. It was wellknown that the $\mathrm{Fe}_{3} \mathrm{O}_{4}$ nanoparticles tend to attract each other and formed a larger cluster origining from the intrinsic anisotropic dipolar attraction, leading to the loss of superparamagnetism. A silica layer was grown onto the surface of $\mathrm{Fe}_{3} \mathrm{O}_{4}$ nanoparticle under vigorous stirring, in order to protect the $\mathrm{Fe}_{3} \mathrm{O}_{4}$ nanoparticle from aggregation and keep them in a stable status. In addition, surfactant-mediated templating method was introduced to produce sufficient pores with larger volume on the silica layer using CTAB as template, and TMB as pore swelling agents. Figure $2 a$ showed the TEM images of the $\mathrm{Fe}_{3} \mathrm{O}_{4} /$ mesoporous $\mathrm{SiO}_{2}$ with the size of nearly $100 \mathrm{~nm}$. Plenty of pores were distributed in the entire silica layer. On the contrary, rare pores were found from Fig. $2 b$, which was obtained by a traditional stöber method without the assistance of CTAB and TMB. The enlarged pores were beneficial to the increment of molecule-loading capacity and the penetrance of irradiation light, as well as the improvement of stability of the nanoplatform. Furthermore, the MPA-stabilized Mn-doped ZnS/ZnS quantum dots were linked onto the surface of the silica shell based on the electrostatic interaction between sulfydryl groups from the MPA and amino groups from the APS. Figure $2 c$ presented the TEM image of the $\mathrm{Fe}_{3} \mathrm{O}_{4} / \mathrm{Mn}$-doped $\mathrm{ZnS} / \mathrm{ZnS}$ nanoplatform. The surface of the nanoplatform got much rougher than the $\mathrm{Fe}_{3} \mathrm{O}_{4}$ /mesoporous $\mathrm{SiO}_{2}$, due to the modification of APS and the decoration of quantum dots. Due to the nontoxicity of the quantum dots and excellent biocompatibility of the nanoplatform, there was no need to coat another $\mathrm{SiO}_{2}$ layer onto the surface of the nanoplatform, which was supior to the previous reports. 
Figure 3a-3c showed wide-angle X-ray diffraction patterns of $\mathrm{Fe}_{3} \mathrm{O}_{4}$ nanoparticle, $\mathrm{Fe}_{3} \mathrm{O}_{4} /$ mesoporous $\mathrm{SiO}_{2}$ and $\mathrm{Fe}_{3} \mathrm{O}_{4} / \mathrm{Mn}$-doped $\mathrm{ZnS} / \mathrm{ZnS}$ nanoplatform. In Fig. 3a, the diffraction pattern indicated the cubic structure of $\mathrm{Fe}_{3} \mathrm{O}_{4}$ nanoparticle, represented by the peaks (20) located at $30^{\circ}, 35.4^{\circ}, 43^{\circ}, 53.4^{\circ}, 56.9^{\circ}$, and $62.5^{\circ}$ corresponding to [220], [311], [400], [422], [511], and [440] planes, which was similar with the previous reports ${ }^{[31,32]}$. According to the Sherrer's formula, the average size of the $\mathrm{Fe}_{3} \mathrm{O}_{4}$ nanoparticle was calculated at nearly $11 \mathrm{~nm}$, which was consistent with their TEM image. In the X-ray diffraction pattern of $\mathrm{Fe}_{3} \mathrm{O}_{4} /$ mesoporous $\mathrm{SiO}_{2}$ (Fig. 3b), a new distinct diffraction peak located at around $23^{\circ}$ appeared apart from the main peak of $\mathrm{Fe}_{3} \mathrm{O}_{4}$ nanoparticle, implying that the silica shell was efficiently coated onto the surface of the $\mathrm{Fe}_{3} \mathrm{O}_{4}$. As shown in Fig. 3c, the X-ray diffraction pattern of the $\mathrm{Fe}_{3} \mathrm{O}_{4} / \mathrm{Mn}$-doped $\mathrm{ZnS} / \mathrm{ZnS}$ nanoplatform was similar with these of $\mathrm{Fe}_{3} \mathrm{O}_{4}$ /mesoporous $\mathrm{SiO}_{2}$, and only tiny weak diffraction peaks of Mn-doped ZnS/ZnS quantum dots appeared due to little mass of quantum dots relative to that of $\mathrm{Fe}_{3} \mathrm{O}_{4} /$ mesoporous $\mathrm{SiO}_{2}$.

The magnetic behaviors of the $\mathrm{Fe}_{3} \mathrm{O}_{4}$ nanoparticle and nanoplatform were measured by vibrating sample magnetometer at room temperature $(300 \mathrm{~K})$ in Fig. 4 . It was demonstrated that all the samples exhibited excellent superparamagnetism due to the absence of remanence and coercivity. From these dates, the magnetic saturation of the $\mathrm{Fe}_{3} \mathrm{O}_{4}$ nanoparticle was calculated to $55 \mathrm{emu} / \mathrm{g}$, while that of the nanoplatform was measured to $32 \mathrm{emu} / \mathrm{g}$, which was lower than the Fe304 nanoparticle. The reason was that the mesoporous $\mathrm{SiO}_{2}$ layer and quantum dots contributed major portion of the gross mass of the nanoplatform. In spite of the certain reduction of the magnetic saturation, the value of $32 \mathrm{emu} / \mathrm{g}$ for the nanoplatform was high enough for the application in magnetic separation. Furthermore, separation test was conducted in Fig. 4(inset), demonstrating that the nanoplatform was easily dragged onto the targeted side by an external magnet in 5 minutes.

Figure 5 shows the fluorescence spectra of the Mn-doped $\mathrm{ZnS} / \mathrm{ZnS}$ quantum dots and the nanoplatform samples. The concentration of the quantum dots in the two samples was set at a same level in order to discuss the effect from the structural change. The individual Mn-doped ZnS/ZnS quantum dots had an emission peak at $571 \mathrm{~nm}$ with a narrow FWHM (full width at half maximum). For the nanoplatform sample, a slight decrease in the fluorescence spectra was observed, which was reported in the previous literature ${ }^{[33,34]}$. There are two reasons to explain this phenomenon. One reason is that surface state of the quantum dots was changed from the starting thiol groups $(-\mathrm{SH})$ to be chained onto the $\mathrm{Fe}_{3} \mathrm{O}_{4}$ /mesoporous $\mathrm{SiO}_{2}$. Another reason is that the $\mathrm{Fe} 3 \mathrm{O} 4$ nanoparticle would absorb the light launching from the quantum dots, named as energy transfer, leading to the reduction of the fluorescence intensity. In addition, a tiny red-shift (about $5 \mathrm{~nm}$ ) on the emission peak of the nanoplatform sample was observed due to the increment of dielectric constant.

Quantum dots had been verified be ideal labeling probes for biomedical imaging. However, the traditional quantum dots decorated by organic molecule must be treated with hydrophilic group to ensure their biocompatibility, leading to the decrease of the fluorescent intensity and structural stability. In addition to be non-toxic, the surface of the designed nanoplatform was functioned with hydrophilic folic 
acid,effectively avoiding the problem of poor water solubility. After transfection 24 hours with the nanoplatforms, Fig. $6 a$ and $6 \mathrm{~b}$ showed the morphology features of the breast cancer cell (MB-MBA) with the same vision under bright-field and dark-field, respectively. Orange light emitted from the cancer cells could be easily detected from the fluorescent image (Fig. 6a), demonstrating that the nanoplatform was effectively uptaken by the cancer cells. Form the bright-field image (Fig. 6b), the entire cellular outline was in full accord with the fluorescence image. Besides, the shape of the cancer cells still maintained fusiform-like after uptaking the nanoplatform, suggesting that the cancer cell had good cell viability and the nanoplatform had no damage to the cancer cells. These results revealed that the nanoplatform was a good alternative labeling probe in biomedical images.

It was well-known that the nanostructure with NIR (near-infrared) absorption had gain tremendous attention, because the NIR light possessed the deep penetration into tissue and no damage to normal tissues and organs. It was necessary to evaluate the potential capability of the nanoplatform for the photothermal therapy before their using in vitro. As shown in Fig. 7 displaying the curves of the temperature versus time, the laser irradiation $\left(808 \mathrm{~nm}, 1 \mathrm{~W} / \mathrm{cm}^{2}\right)$ was employed to study the photothermal effect of the nanoplatform samples with different concentration. The real-time temperature of the sample was monitored by a thermoelectric thermometer, and every date was recorded by the average of five consecutive measurements. It was known that the cancer cells would lose activity when treated with the temperature of $42^{\circ} \mathrm{C}$ for $15-60$ minutes or over $50^{\circ} \mathrm{C}$ for $4-6$ minutes. As shown in Fig. 7, the higher concentration of the nanoplatform sample, the higher changed temperature increased. For example, the raised temperature of the nanoplatform sample could reach $44^{\circ} \mathrm{C}$ from $25^{\circ} \mathrm{C}$ with the concentration of $2 \mathrm{mg} / \mathrm{mL}$ after irradiation for 15 minutes, which was high enough to destroy the targeted cancer cell. As a control sample, the temperature of the PBS solution raised by only $3.7^{\circ} \mathrm{C}$.

To verify the feasibility of the nanoplatform in destroying cancer cells, the nanoplatform with the concentration of $2 \mathrm{mg} / \mathrm{mL}$ was utilized to treat the cancer cell under laser irradiation $\left(808 \mathrm{~nm}, 1 \mathrm{~W} / \mathrm{cm}^{2}\right)$. After incubated with the nanoplatform for 2 hours, the cancer cells were treated with the laser irradiation for 10 minutes. Then the cancer cell was stained, and evaluated the cell viability. As shown in Fig. 8a, over $98 \%$ of the cancer cells were killed by the treatment of nanoplatform and laser irradiation. In control sample, after incubated with the nanoplatform but without the laser irradiation, the viability of cancer cells was close to be $100 \%$ in Fig. $8 \mathrm{~b}$, which manifested the nanoplatform had no intrinsic toxicity. These study demonstrated that the nanoplatform had the ability to induce the targeted cancer cell apoptosis under the laser irradiation.

\section{Conclusions}

In conclusion, we successfully synthetized ferroferric oxide/quantum dots nanoplatforms and demonstrated their capability for fluorescent labeling and photothermal therapy for cancer cells. The nanoplatform was prepared in terms of layer-by-layer method using $\mathrm{Fe}_{3} \mathrm{O}_{4}$ nanoparticle as the core and mesoporous $\mathrm{SiO}_{2}$ as the protective shell, then loaded with nontoxic Mn-doped $\mathrm{ZnS} / \mathrm{ZnS}$ quantum dots. Notably, in order to lower mass and promote the absorption of light, a template method was employed to 
generate plenty of pores on the silica shell. Due to the decoration of quantum dots, the nanoplatform possessed strong fluorescence behavior, thus exhibiting the potential as fluorescence labeling agents for the targeted cancer cells. Rooting in the superparamagnetism the $\mathrm{Fe}_{3} \mathrm{O}_{4}$ nanoparticle, the nanoplatform showed excellent magnetic separation. Besides, the photothermal experiments in vitro show that the nanoplatform possessed no toxicity and the capability to ablate the targeted cancer cells under laser irradiation. These all-in-one nanoplatforms are expected to be used in the clinical application of cancer detection and therapy.

\section{Declarations}

\section{Acknowledgements}

This work was supported by the National Natural Science Foundation of China (No. 11704237, 51872279, and U1704145) and the Henan Provincial Natural Science Foundation (No.202102310540, and No. 192102210199).

\section{Data availability}

The raw/processed data required to reproduce these findings cannot be shared at this time due to technical or time limitations.

\section{References}

[1] B. Getiren, Z. Ciplak, C. Gokalp and N. Yildiz, NIR-responsive Fe304@PPy nanocomposite for efficient potential use in photothermal therapy, Journal of Applied Polymer Science. 137 (2020) e49343.

[2] Q. Zhang, W. Wang, M. Zhang, F. Wu, T. Zheng, B. Sheng, Y. Liu, J. Shen, N. Zhou and Y. Sun, A theranostic nanocomposite with integrated black phosphorus nanosheet, Fe304@MnO2-doped upconversion nanoparticles and chlorin for simultaneous multimodal imaging, highly efficient photodynamic and photothermal therapy, Chemical Engineering Journal. 391 (2020) 123525.

[3] G. Guan, X. Wang, B. Li, W. Zhang, Z. Cui, X. Lu, R. Zou and J. Hu, "Transformed" Fe3S4 tetragonal nanosheets: a high-efficiency and body-clearable agent for magnetic resonance imaging guided photothermal and chemodynamic synergistic therapy, Nanoscale. 10 (2018) 17902-17911.

[4] L.-S. Lin, T. Huang, J. Song, X.-Y. Ou, Z. Wang, H. Deng, R. Tian, Y. Liu, J.-F. Wang, Y. Liu, G. Yu, Z. Zhou, S. Wang, G. Niu, H.-H. Yang and X. Chen, Synthesis of Copper Peroxide Nanodots for H2O2 Self-Supplying Chemodynamic Therapy, Journal of the American Chemical Society. 141 (2019) 9937-9945.

[5] Z. Tang, Y. Liu, M. He and W. Bu, Chemodynamic Therapy: Tumour Microenvironment-Mediated Fenton and Fenton-like Reactions, Angewandte Chemie-International Edition. 58 (2019) 946-956. 
[6] L. Ga, J. Ai and Y. Wang, AS1411-Templated Fluorescent Cu Nanomaterial's Synthesis and Its Application to Detecting Melamine, Journal of Chemistry. 2020 (2020) 4067578.

[7] R. Sun, L. Yin, S. Zhang, L. He, X. Cheng, A. Wang, H. Xia and H. Shi, Simple Light-Triggered Fluorescent Labeling of Silica Nanoparticles for Cellular Imaging Applications, Chemistry-a European Journal. 23 (2017) 13893-13896.

[8] X. Hou, X. Wang, R. Liu, H. Zhang, X. Liu and Y. Zhang, Facile synthesis of multifunctional Fe304@SiO(2)n@Au magneto-plasmonic nanoparticles for MR/CT dual imaging and photothermal therapy, Rsc Advances. 7 (2017) 18844-18850.

[9] C. Li, T. Chen, I. Ocsoy, G. Zhu, E. Yasun, M. You, C. Wu, J. Zheng, E. Song, C. Z. Huang and W. Tan, Gold- Coated Fe 304 Nanoroses with Five Unique Functions for Cancer Cell Targeting, Imaging, and Therapy, Advanced Functional Materials. 24 (2014) 1772-1780.

[10] Y. Li, X. Liu, X. Nie, W. Yang, Y. Wang, R. Yu and J. Shui, Multifunctional Organic-Inorganic Hybrid Aerogel for Self-Cleaning, Heat-Insulating, and Highly Efficient Microwave Absorbing Material, Advanced Functional Materials. 29 (2019) 1807624.

[11] W. Wang, C. Hao, M. Sun, L. Xu, C. Xu and H. Kuang, Spiky Fe304@Au Supraparticles for Multimodal In Vivo Imaging, Advanced Functional Materials. 28 (2018) 1800310.

[12] Y. Hu, L. Meng, L. Niu and Q. Lu, Facile Synthesis of Superparamagnetic Fe304@polyphosphazene@Au Shells for Magnetic Resonance Imaging and Photothermal Therapy, Acs Applied Materials \& Interfaces. 5 (2013) 4586-4591.

[13] D. Liu, L. Ma, L. Liu, L. Wang, Y. Liu, Q. Jia, Q. Guo, G. Zhang and J. Zhou, PolydopamineEncapsulated Fe304 with an Adsorbed HSP70 Inhibitor for Improved Photothermal Inactivation of Bacteria, Acs Applied Materials \& Interfaces. 8 (2016) 24455-24462.

[14] Y. Wang, X. Pang, J. Wang, Y. Cheng, Y. Song, Q. Sun, Q. You, F. Tan, J. Li and N. Li, Magneticallytargeted and near infrared fluorescence/magnetic resonance/photoacoustic imaging-guided combinational anti-tumor phototherapy based on polydopamine-capped magnetic Prussian blue nanoparticles, Journal of Materials Chemistry B. 6 (2018) 2460-2473.

[15] P. Kharey, S. B. Dutta, M. Manikandan, I. A. Palani, S. K. Majumder and S. Gupta, Green synthesis of near-infrared absorbing eugenate capped iron oxide nanoparticles for photothermal application, Nanotechnology. 31 (2020) 095705.

[16] Y. Chen, M. Xu, Y. Guo, K. Tu, W. Wu, J. Wang, X. Tong, W. Wu, L. Qi and D. Shi, Targeted chimera delivery to ovarian cancer cells by heterogeneous gold magnetic nanoparticle, Nanotechnology. 28 (2017) 025101. 
[17] M. J. Chen, H. Shen, X. Li and H. F. Liu, Facile synthesis of oil-soluble Fe304 nanoparticles based on a phase transfer mechanism, Applied Surface Science. 307 (2014) 306-310.

[18] F. Li, Y. Wang and T. Wang, Synthesis of Fe304 particle-chain microwires in applied magnetic field, Journal of Solid State Chemistry. 180 (2007) 1272-1276.

[19] S. Photocatalytic degradation of aniline by magnetic nanomaterials Fe304@SiO2@BiOKhammar, N. Bahramifar and $\mathrm{H}$. Younesi, Optimization using the response surface methodology for adsorption of polychlorinated biphenyls (PCBs) from transformer oil by magnetic CMCD-Fe304@SiO2 nanoparticles, Materials Chemistry and Physics. 252 (2020) 123195.

[20] M. Shao, F. Ning, J. Zhao, M. Wei, D. G. Evans and X. Duan, Preparation of Fe304@SiO2@Layered Double Hydroxide Core-Shell Microspheres for Magnetic Separation of Proteins, Journal of the American Chemical Society. 134 (2012) 1071-1077.

[21] R. H. Yellow emitting Fe304/ZnS hybrid: A probe for in-vitro dermatogJin, Z. X. Qiu, W. Cheng and X. L. Jin, Photocatalytic degradation of aniline by magnetic nanomaterials Fe304@SiO2@Bi01.8 center dot 0.04H(2)O/Ag3P04, Chemical Physics Letters. 755 (2020) 137747.

[22] J. Kim, H. S. Kim, N. Lee, T. Kim, H. Kim, T. Yu, I. C. Song, W. K. Moon and T. Hyeon, Multifunctional Uniform Nanoparticles Composed of a Magnetite Nanocrystal Core and a Mesoporous Silica Shell for Magnetic Resonance and Fluorescence Imaging and for Drug Delivery, Angewandte Chemie-International Edition. 47 (2008) 8438-8441.

[23] D.-W. Wang, X.-M. Zhu, S.-F. Lee, H.-M. Chan, H.-W. Li, S. K. Kong, J. C. Yu, C. H. K. Cheng, Y.-X. J. Wang and K. C.-F. Leung, Folate-conjugated Fe304@SiO2@gold nanorods@mesoporous SiO2 hybrid nanomaterial: a theranostic agent for magnetic resonance imaging and photothermal therapy, Journal of Materials Chemistry B. 1 (2013) 2934-2942.

[24] M. Wang, C.-C. Mi, W.-X. Wang, C.-H. Liu, Y.-F. Wu, Z.-R. Xu, C.-B. Mao and S.-K. Xu, Immunolabeling and NIR-Excited Fluorescent Imaging of HeLa Cells by Using NaYF4:Yb,Er Upconversion Nanoparticles, Acs Nano. 3 (2009) 1580-1586.

[25] Y. Y. Zhao, M. Song, X. G. Yang, J. Yang, C. Y. Du, G. N. Wang, J. W. Yi, G. Y. Shan, D. S. Li, L. Liu, D. M. Yan, Y. X. Li and X. J. Liu, Amorphous Ag2-xCuxS quantum dots: "all-in-one" theranostic nanomedicines for near-infrared fluorescence/photoacoustics dual-modal-imaging-guided photothermal therapy, Chemical Engineering Journal. 399 (2020) 125777.

[26] A. K. H. Cheng, H. Su, A. Wang and H.-Z. Yu, Aptamer-Based Detection of Epithelial Tumor Marker Mucin 1 with Quantum Dot-Based Fluorescence Readout, Analytical Chemistry. 81 (2009) 6130-6139.

[27] G. Jie, L. Wang, J. Yuan and S. Zhang, Versatile Electrochemiluminescence Assays for Cancer Cells Based on Dendrimer/CdSe-ZnS-Quantum Dot Nanoclusters, Analytical Chemistry. 83 (2011) 3873-3880. 
[28] H. Nie, M. Li, Q. Li, S. Liang, Y. Tan, L. Sheng, W. Shi and S. X.-A. Zhang, Carbon Dots with Continuously Tunable Full-Color Emission and Their Application in Ratiometric pH Sensing, Chemistry of Materials. 26 (2014) 3104-3112.

[29] N. Yin, L. Liu, P. Li and S. Zhao, Tunable excitation properties of ZnCdS:Mn/ZnS quantum dots for cancer imaging, Luminescence. 33 (2018) 630-635.

[30] N. Q. Yin, P. Wu, T. H. Yang and M. Wang, Preparation and study of a mesoporous silica-coated Fe304 photothermal nanoprobe, Rsc Advances. 7 (2017) 9123-9129.

[31] S. N. A. Keivani, M. Naderi and G. Amoabediny, Superparamagnetic plasmonic nanocomposites: Synthesis and characterization studies, Chemical Engineering Journal. 264 (2015) 66-76.

[32] S. Shen, S. Wang, R. Zheng, X. Zhu, X. Jiang, D. Fu and W. Yang, Magnetic nanoparticle clusters for photothermal therapy with near-infrared irradiation, Biomaterials. 39 (2015) 67-74.

[33] J.-M. Lei, X.-L. Xu, L. Liu, N.-Q. Yin and L.-X. Zhu, Preparation and Characterization of Bimodal Magnetofluorescent Nanoprobes for Biomedical Application, Chinese Physics Letters. 29 (2012) 097803.

[34] N.-Q. Yin, L. Liu, J.-M. Lei, Y.-S. Liu, M.-G. Gong, Y.-Z. Wu, L.-X. Zhu and X.-L. Xu, Preparation and characterization of nontoxic magnetic-luminescent nanoprobe, Chinese Physics B. 21 (2012) 116101.

\section{Figures}



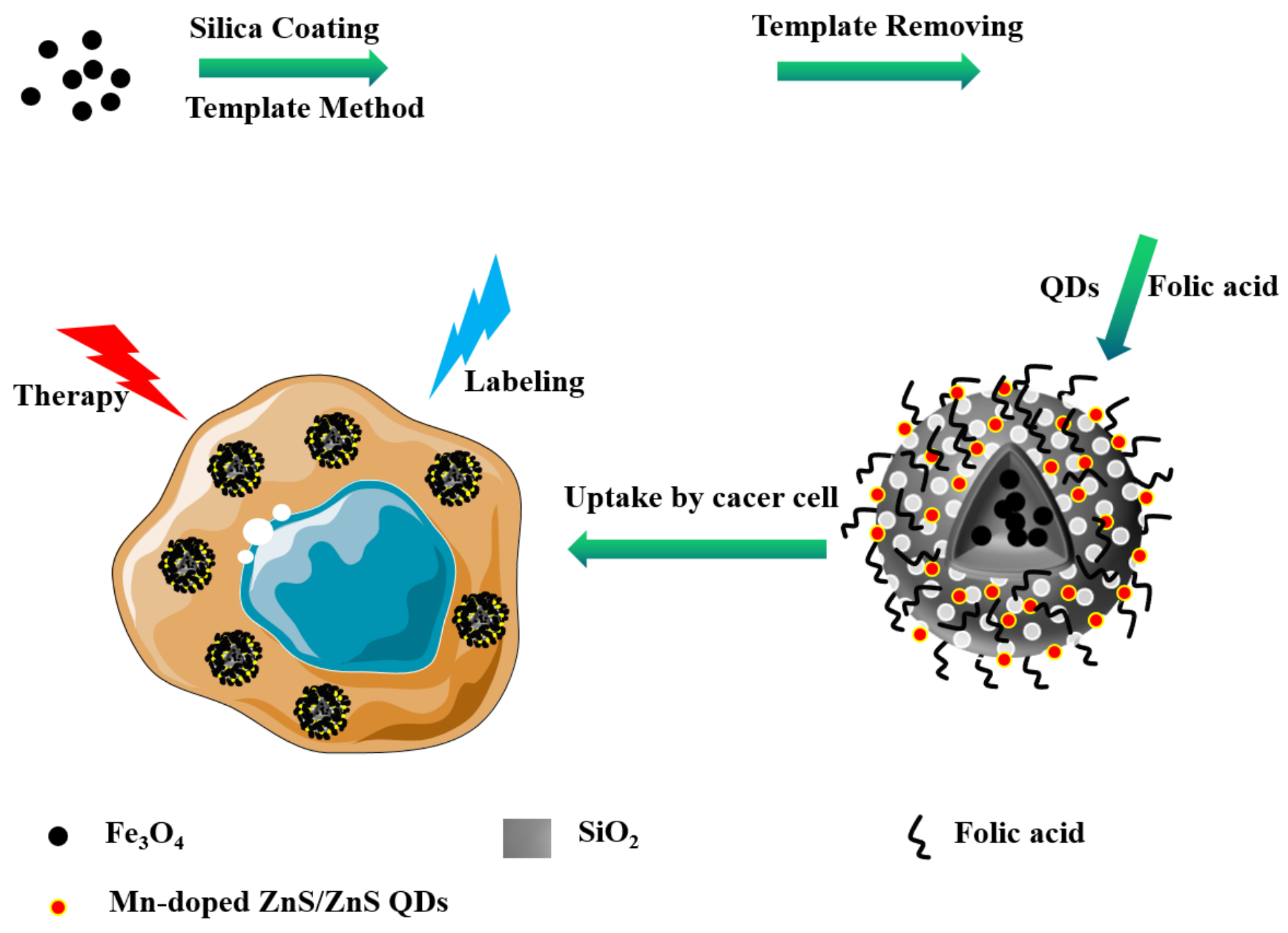

Figure 1

Schematic of the synthetic process of nontoxic Fe304/Mn-doped ZnS/ZnS nanoplatforms

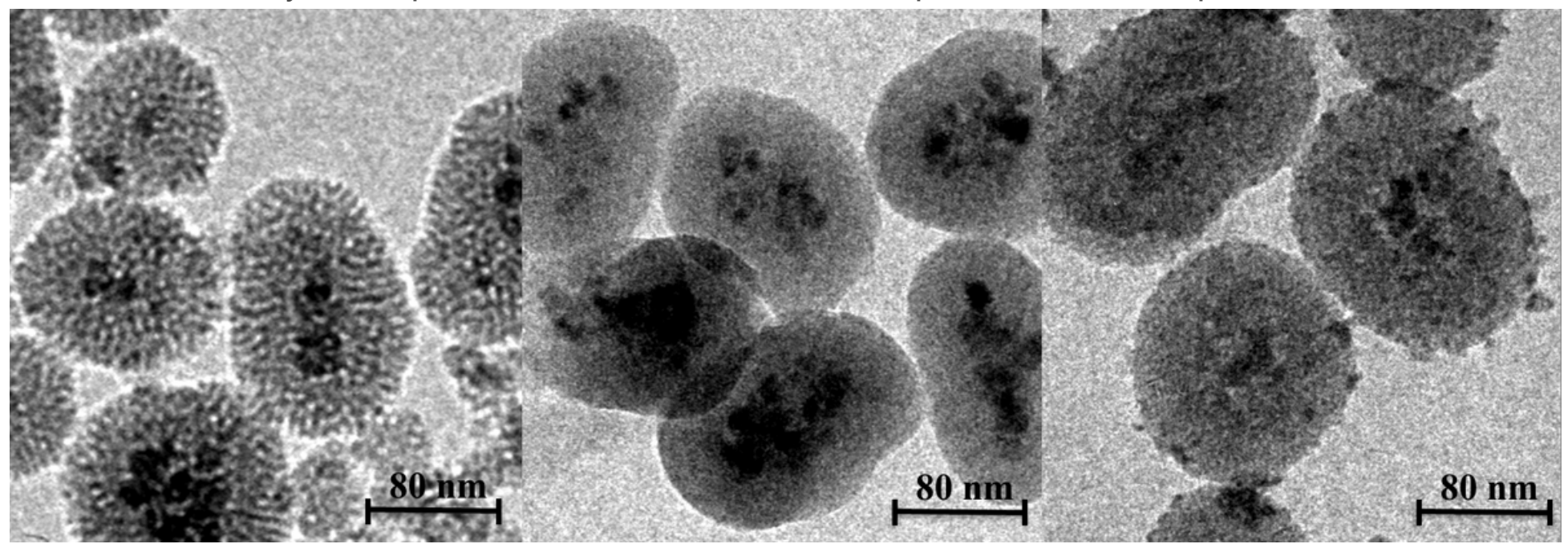

Figure 2 
TEM images of (a) Fe304/mesoporous SiO2 structure, (b) Fe304/SiO2 structure and (c) Fe304/Mndoped ZnS/ZnS nanoplatform

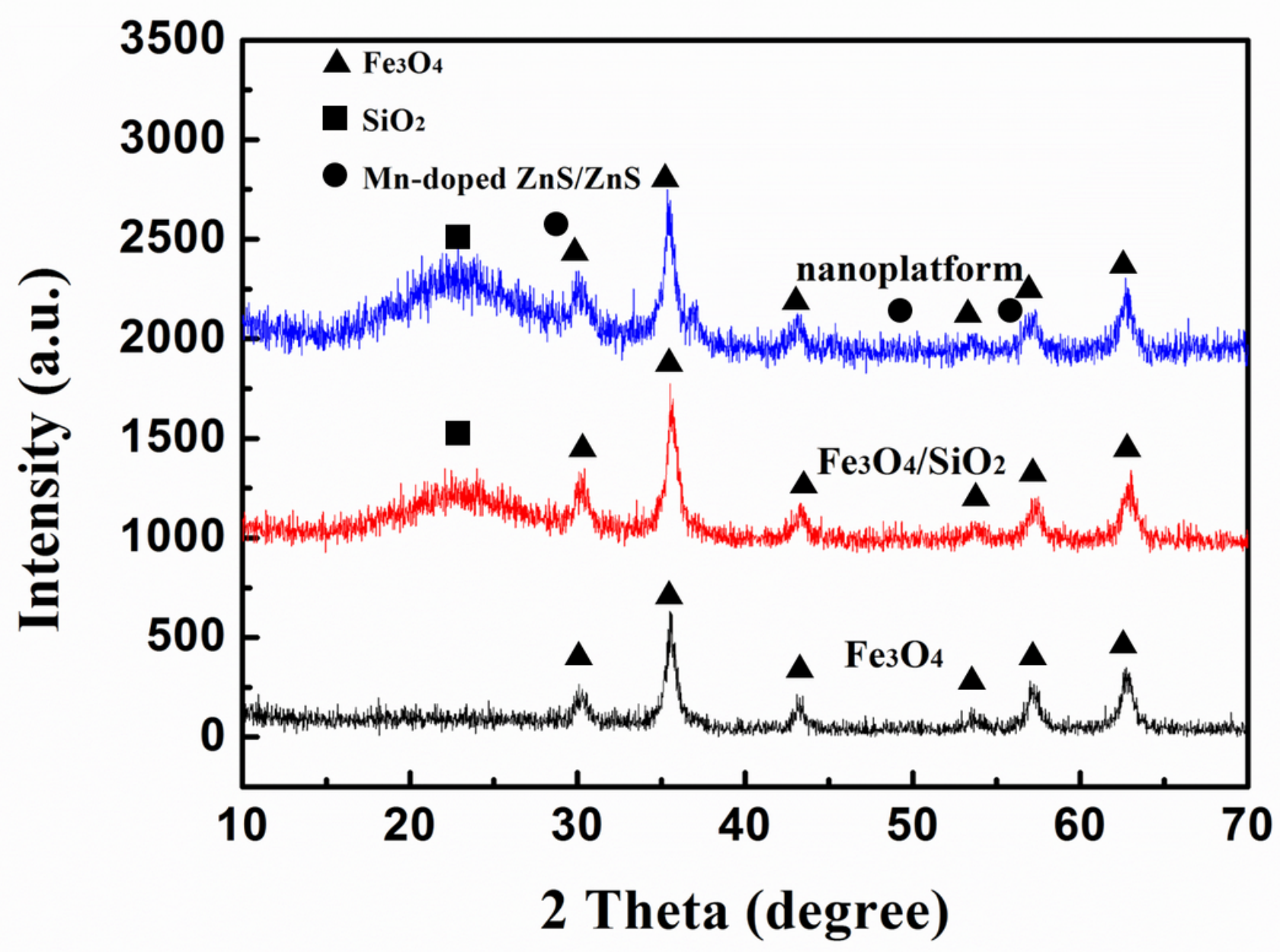

Figure 3

Power X-ray diffraction (XRD) patterns of (a) Fe304, (b) Fe304/mesoporous SiO2 structure, (c) Fe304/Mn-doped ZnS/ZnS nanoplatform 


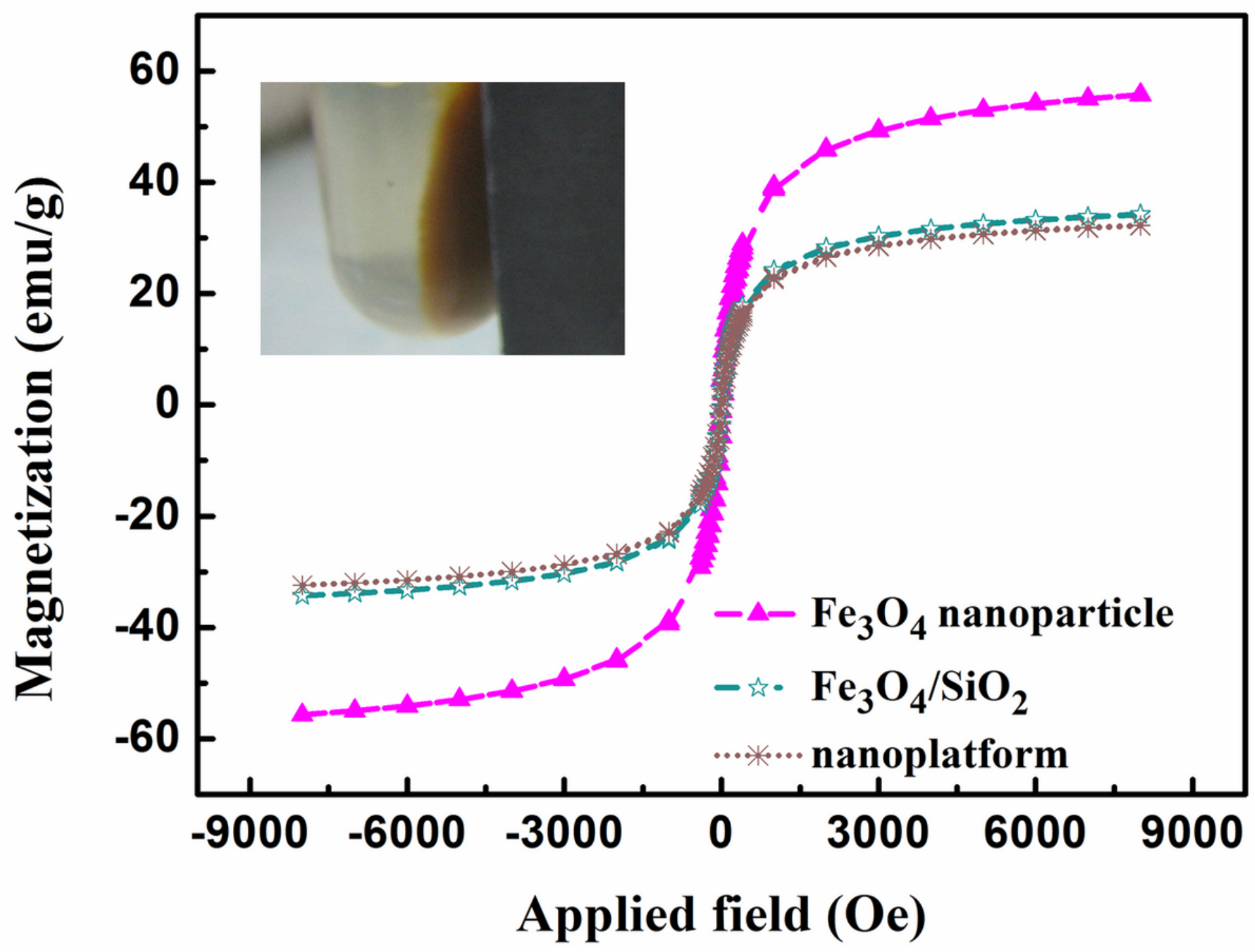

Figure 4

Magnetic hysteresis curve of (a) Fe304, (b) Fe304/mesoporous SiO2 structure, (c) Fe304/Mn-doped $\mathrm{ZnS} / \mathrm{ZnS}$ nanoplatform. Inset is the photographic image of nanoplatform dragged an extern magnet under fluorescence microscope. 


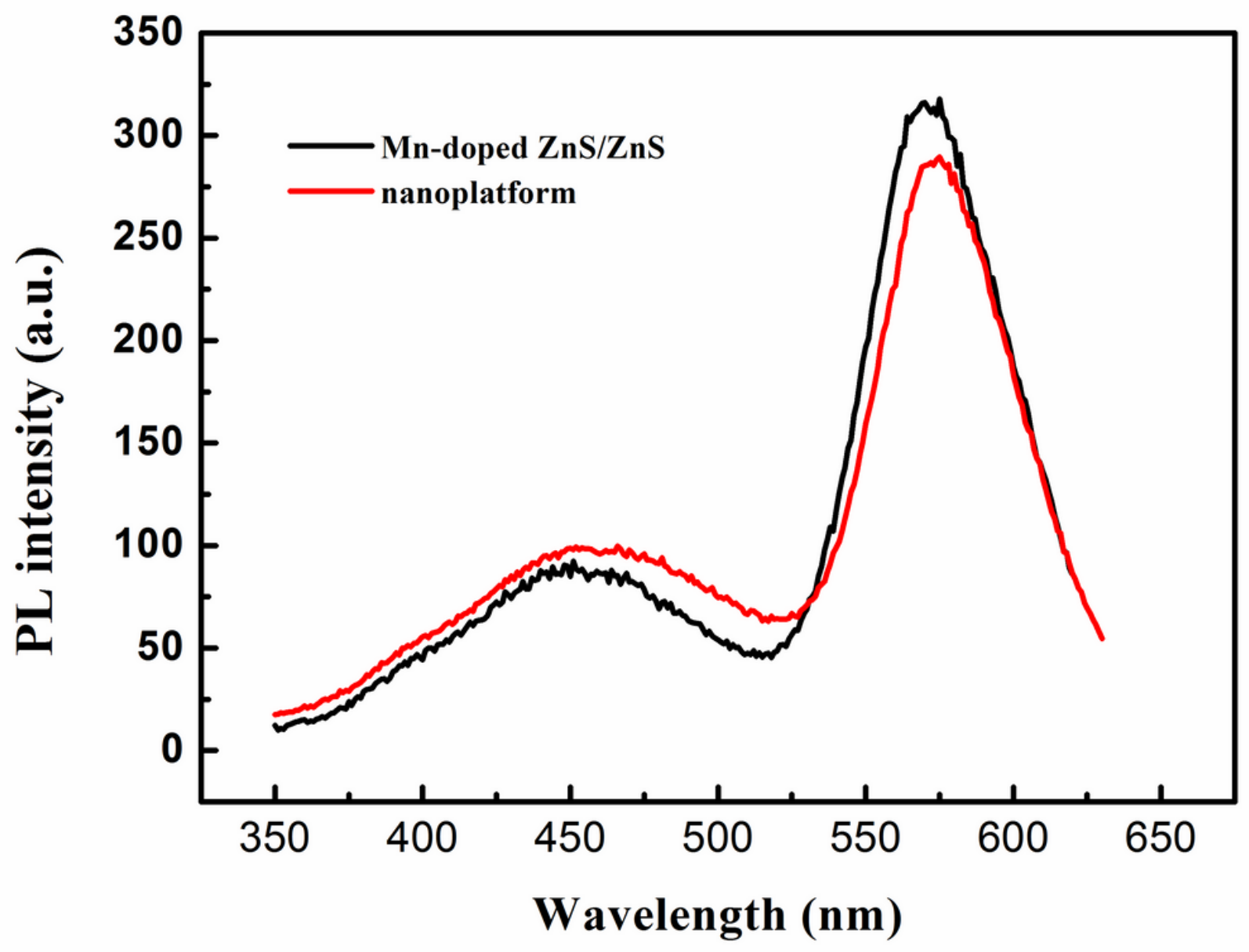

Figure 5

Fluorescence spectra of the Mn-doped ZnS/ZnS quantum dots and the nanoplatform.

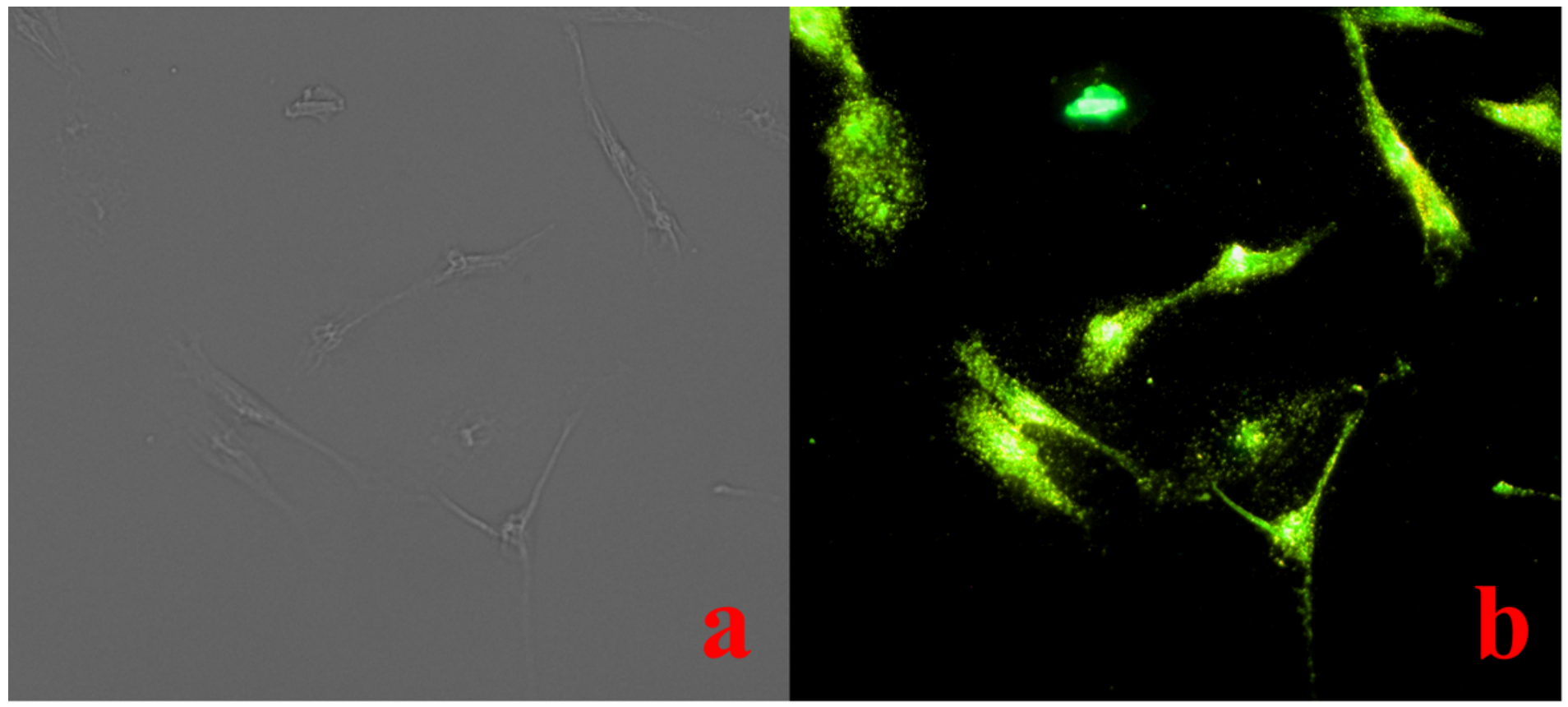


Figure 6

Fluorescent mapping of the cancer cells under (a) bright field and (b) dark field.

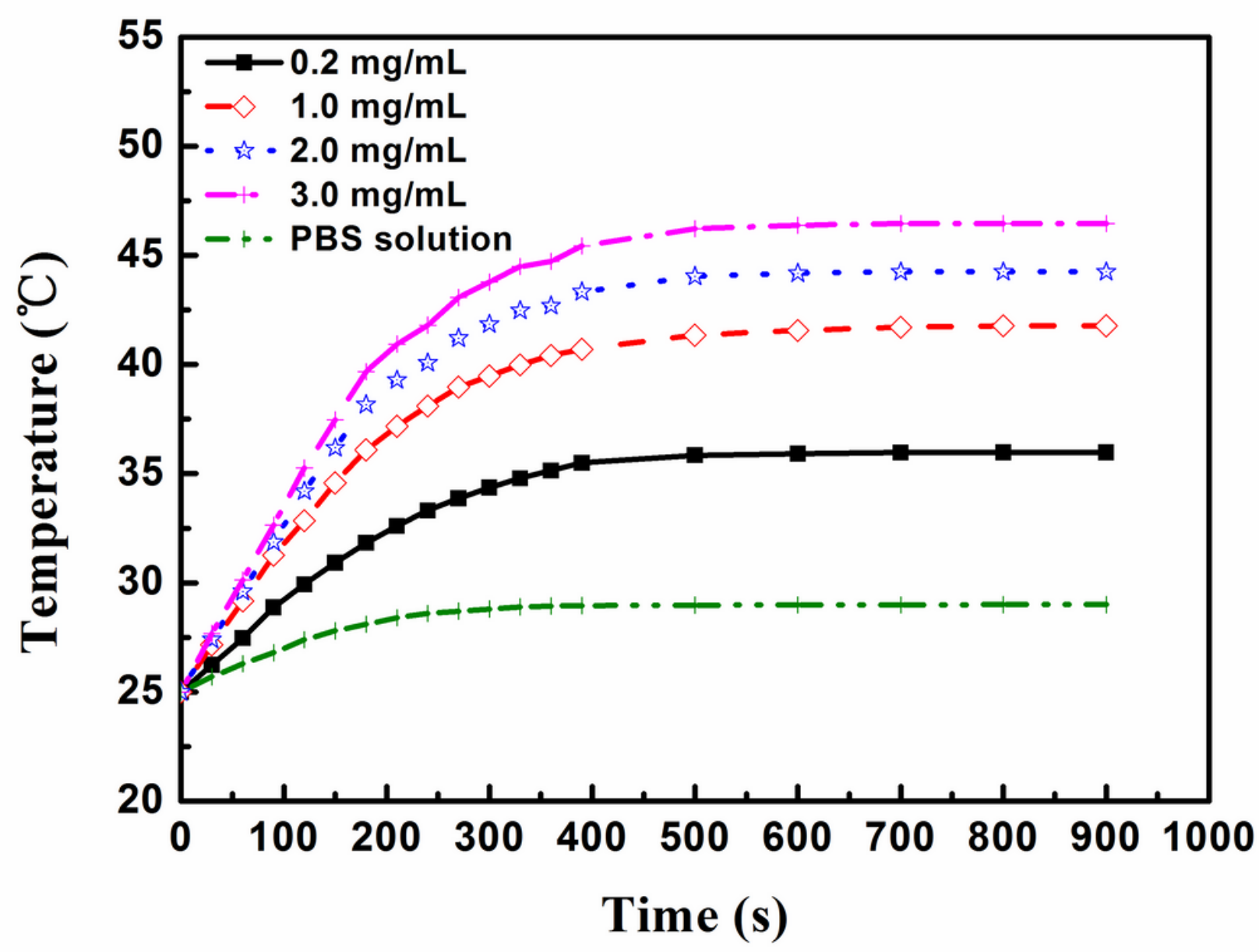

Figure 7

Temperature change curves of the Fe304/Mn-doped $\mathrm{ZnS} / \mathrm{ZnS}$ nanoplatform with different concentration $(0.2 \mathrm{mg} / \mathrm{mL}, 1.0 \mathrm{mg} / \mathrm{mL}, 2.0 \mathrm{mg} / \mathrm{mL}$, and $3.0 \mathrm{mg} / \mathrm{mL})$ under $808 \mathrm{~nm}$ laser irradiation with the power density of $1.0 \mathrm{~W} / \mathrm{cm} 2$ for 15 minutes. 


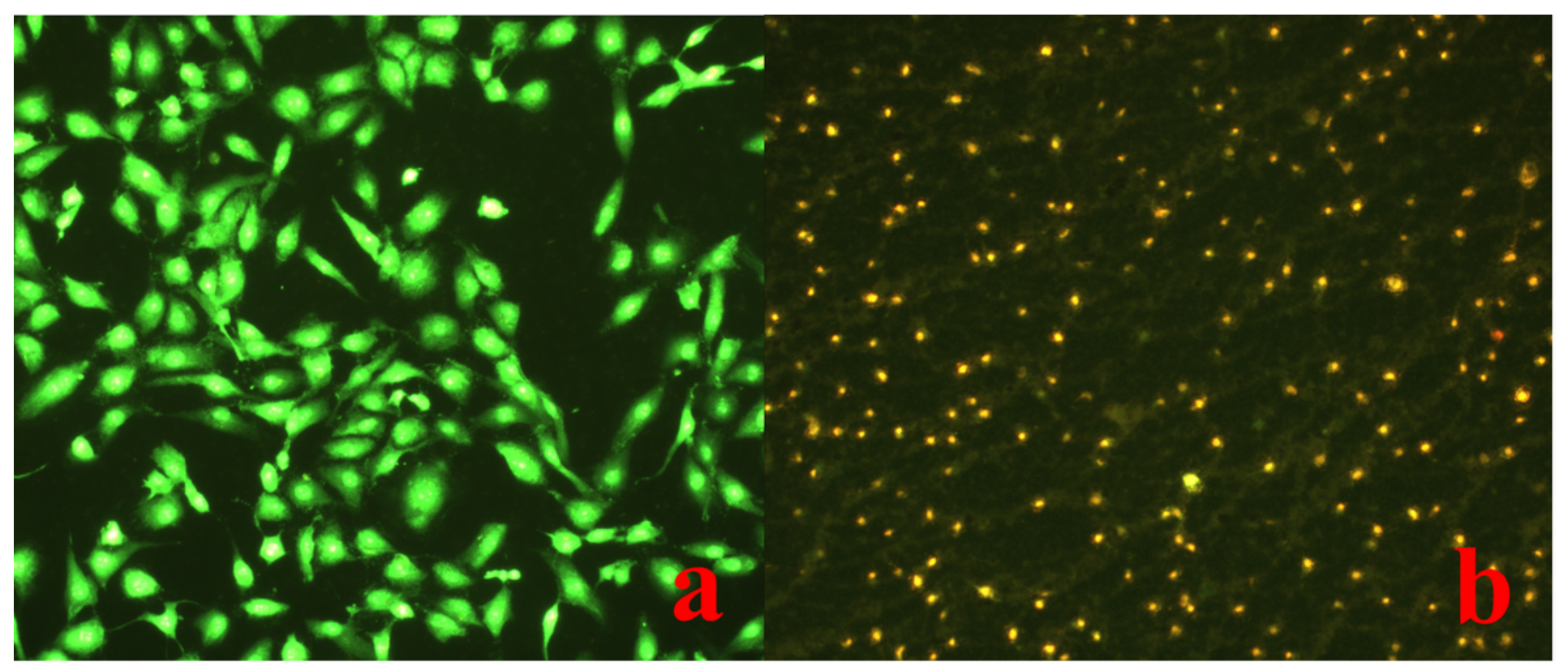

Figure 8

Fluorescence images of the cancer cells with treated with (a) Fe304/Mn-doped ZnS/ZnS nanoplatform, (b) PBS solution. The cancer cells were co-stained by Calcian Am (green, live cells) and propidium iodide (red, dead cells). 